\title{
Images matérielles et images immatérielles dans les sermons de saint Bernardin de Sienne
}

Immagini materiali e immagini immateriali nelle prediche di San Bernardino da Siena

Material and Immaterial Images in the Sermons of Saint Bernardine of Siena

Serge Stolf

\section{(2) OpenEdition}

\section{Journals}

Édition électronique

URL : http://journals.openedition.org/cei/5913

DOI : $10.4000 /$ cei.5913

ISSN : 2260-779X

Éditeur

UGA Éditions/Université Grenoble Alpes

Édition imprimée

ISBN : 978-2-37747-159-1

ISSN : 1770-9571

Référence électronique

Serge Stolf, «Images matérielles et images immatérielles dans les sermons de saint Bernardin de Sienne ", Cahiers d'études italiennes [En ligne], 29 | 2019, mis en ligne le 30 septembre 2019, consulté le 27 mars 2021. URL : http://journals.openedition.org/cei/5913 ; DOI : https://doi.org/ 10.4000/cei.5913

Ce document a été généré automatiquement le 27 mars 2021.

(c) ELLUG 


\title{
Images matérielles et images immatérielles dans les sermons de saint Bernardin de Sienne
}

\author{
Immagini materiali e immagini immateriali nelle prediche di San Bernardino \\ da Siena \\ Material and Immaterial Images in the Sermons of Saint Bernardine of Siena
}

\section{Serge Stolf}

"Più muovono gli essempli di quello che si vede, che le parole che si dicono ${ }^{1}$." Bernardin de Sienne adapte librement une citation de Grégoire le $\mathrm{Grand}^{2}$ pour affirmer que l'exemple visible convainc parfois mieux que des discours. Cette affirmation s'inscrit certes dans un contexte particulier - l'exemple de ses bonnes vie et mœurs que chacun devrait donner -, mais fait aussi appel à la dimension performative du sermon, à savoir le movere, "l'émotion» mais aussi «la motion»: en d'autres termes, sa capacité à mettre en mouvement un agir, mais également à imprimer assez durablement la mémoire de l'auditeur-spectateur qui réactivera celle-ci pour un «faire». En outre, la citation met en tension le voir et le dire, car l'exemple n'est jamais qu'un récit, et la parole, en faisant voir, possède donc cette potentialité de susciter des images efficientes dans le but de corriger des comportements altérés, de proposer des comportements pratiques ajustés à une dimension de vie morale et spirituelle. Dans les limites de ce court article, je m'intéresserai à l'emploi, dans le discours homilétique, d'images matérielles ou sensibles, ou « corporelles ", selon la terminologie de l'époque, et d'images immatérielles, ou mentales, suscitées par les images sensibles (ou représentations) et verbales ${ }^{3}$ - métaphores, allégories - qui donnent à voir sous les yeux par un effet d'enargeia, c'est-à-dire d'évidence visuelle, selon la terminologie de la Rhétorique d'Aristote ${ }^{4}$. Horace, dans son Art poétique, déclarait : « Les esprits sont moins vivement frappés par ce qui est confié à l'oreille / que ce qui est mis sous leurs yeux, témoins sûrs $[. . .]^{5}$.» L'exemple par l'image comme moyen privilégié pour faire « comprendre quelque chose ${ }^{6}$ » à son auditoire constitue un élément fondamental du sermon bernardinien à destination d'un public populaire. J'aborderai brièvement les 
trois points suivants : le premier s'attachera à poser les fondements d'une culture du visuel dans les sermons de Bernardin, le second à donner un certain nombre d'exemples des relations dynamiques établies entre image réelle et image mentale comme outils performatifs, et donc comme outils de mémoire, et le troisième à évoquer la médiation de deux images particulières, la croix et la tablette du Nom de Jésus, cette dernière associant image et écriture.

\section{Importance de la rhétorique visuelle chez Bernardin}

2 Pour aborder le premier point sur l'importance de la rhétorique visuelle mise en œuvre dans les sermons de Bernardin, je citerai préalablement Tommaso da Celano rapportant de saint François d'Assise prêchant: "[...] il faisait de son corps tout entier une langue ${ }^{7}$.» Le même biographe relate que François, invité à prêcher à ses filles de San Damiano, «se fit apporter de la cendre, [qu'] il en dessina un cercle autour de lui sur les dalles et [qu'il] répandit le reste sur sa tête ${ }^{8} »$. Il demeure longtemps ainsi, en silence, puis se lève, déclame "en guise de [à la place d'un] sermon, le psaume Miserere » et sort. « Il leur avait ainsi appris, par son action symbolique, à se considérer comme cendre et poussière ", ajoute Tommaso qui met dans son commentaire l'accent sur l'action avant la parole: c'est par une image efficace, par cette représentation spectaculaire, au sens premier du terme, que François donne à voir un rituel de pénitence et donne à méditer sur le néant de l'homme face à Dieu. Il en obtient un effet immédiat, puisque ces religieuses sont aussitôt remplies de "contrition ». L'image est ainsi chargée d'une force émotionnelle telle que la mémoire pourra la faire surgir à nouveau, comme image mentale réactivant son message ${ }^{9}$.

Bernardin a parfaitement assimilé cette théâtralisation de la parole ("théâtre » est dérivé du verbe grec "regarder, contempler») et du visuel. Ainsi, il intime à son auditoire d'être attentif, dans les termes suivants : «Tu debbi stare attento a udire, per modo che a pena tu non batta l'occhio [senza batter ciglio], mirando in viso il predicatore, e non avere l'occhio bàdolo in quà e là, ma fitto a non mirare in altro lato ${ }^{10}$. " C'est dire que la parole du prédicateur passe, pour être audible, par le regard attaché à sa personne comme image : écouter, c'est aussi voir. C'est par l'image sensible que l'esprit peut connaître, dit saint Thomas d'Aquin dans la Somme, à la suite d'Aristote ${ }^{11}$, ajoutant que « la vue [...] est la faculté la plus spirituelle, le plus parfait de tous les sens et le plus universel ${ }^{12}$ ", mais c'est un théologien franciscain, saint Bonaventure, que cite Bernardin: "Coll'esemplo m'intenderai. Dice Buonaventura: "Plus movent visa, quam audita". Più si tiene a mente quello che altri vede, che quello che altri ode ${ }^{13}$. » Et encore : "Molto è meglio e più lecito il vedere che l'udire, perché la virtù visiva è posta in più alto luogo che l'udito ${ }^{14}$. » L'image (ici, celle que développe l'exemplum) s'imprime davantage dans la mémoire (più si tiene a mente), mais elle offre un meilleur accès à la compréhension (m'intenderai). Les occurrences du verbe vedere (et ses variantes: riguardare, mirare, qui comportent des nuances) sont innombrables dans les sermons de Bernardin.

4 Pour éviter toute ambiguïté dans mon propos, je dois distinguer trois sens dans l'emploi du verbe. Premièrement, celui, concret, de "percevoir par la vue »: «Queste cose voi le vedete tuttodi ${ }^{15} »$; ou bien, renvoyant le public siennois à une image peinte de l'Assomption de la Vierge : «Tutti li Angioli le stanno d'intorno [...] come tu vedi dipento colà su alla Porta a Camollia ${ }^{16}$. " Deuxièmement, le sens abstrait, celui d'imaginer ou de se représenter une chose non présente : «Maria [...] saglie là, dove, se tu la consideri, tu la 
vedrai incoronata come vera imperadrice $\left[. . .{ }^{17}\right.$. " Troisièmement, celui de "prendre connaissance, examiner»: "Va' vede nel Genesis al primo capitolo [...] ${ }^{18}$ " (qu'il va d'ailleurs citer), "voliamo vederle più spiegatamente ${ }^{19}$ ». Ces distinctions, nécessaires, n'excluent pas la contamination des différents sens. En effet, l'appel aux images sensibles comme aux images mentales les distingue sous deux régimes, mais leur confère la même vertu efficiente: le prédicateur leur attribue la même valeur rhétorique. Quant au troisième sens, il pourrait faire l'objet d'une extension - la connaissance procédant par mémorisation d'images - si l'on reprend par exemple la Rhetorica Ad Herennium, attribuée à Cicéron, où il est question de la « mémoire pour les mots $^{20}$ » qui doit trouver des images pour rappeler chaque mot (I, VII, 9). Nous en avons un exemple chez Bernardin, quand il cite le passage de l'Apocalypse où Jean voit un ange descendre du ciel sous des aspects visibles $(10,1-2)$, et qu'il commente : « Là dove tu vedi in queste parole sette proprietà di coloro che vogliono vivare bene ${ }^{21}$. » Le vedere passe par la vision de saint Jean traduite en mots et Bernardin renvoie, là encore, à une perception visuelle du texte comme image : les mots font image.

Pour en terminer avec ce premier point, quelles sont les facultés dont dispose l'homme pour " voir " non seulement les réalités sensibles, mais pour s'ouvrir, par elles, aux réalités immatérielles et spirituelles ? Bernardin, à plusieurs reprises, rappelle le mot du Christ guérissant le sourd-muet: «Ephphetha!» («Ouvre-toi $\left.{ }^{22} ! »\right)$. Il invite l'auditoire et la ville de Sienne à «s'ouvrir» : «O Siena, apre gli occhi, apre l'orecchie a volere udire e intendare e vedere la tua salute ${ }^{23 !}$ » Bernardin insiste conjointement sur l'ouverture du sens de la vue et de l'ouïe (« apreti; apre l'occhio dello intelletto, apre le orecchia $\left.{ }^{24} »\right)$. Il fait appel ailleurs à l'image mentale qu'éveille sa description de l'Ascension de Marie au ciel : «Io mi credo che tu l'hai potuta vedere collo intelletto salire alla gloria, invitata da tutti li spiriti beati $[. . .]^{25}$.» Cette terminologie, Bernardin l'emprunte à toute une tradition médiévale qui prend sa source dans saint Augustin distinguant trois types de visions ${ }^{26}$ : corporelle, spirituelle (imagination), intellectuelle (pour saisir une signification). Saint Bonaventure reprend cette distinction dans son Itinéraire de l'esprit vers Dieu ${ }^{27}$, auquel emprunte Bernardin, ici comme ailleurs où il parle de l'occhio corporale. Cet œil de la connaissance intime et intellectuelle signifie le dépassement de la perception sensible, le passage de l'image sensible à l'appréhension des vérités spirituelles qui sont le vrai but du sermon. Nous verrons également que l'œil et le cœur peuvent confluer en un œil du cœur (ou de l'âme), selon saint Paul ("Qu'il ouvre à sa lumière les yeux de votre cœur $\left.^{28} »\right)$, repris par saint Bernard qui parle de "l'œil intérieur» ou de «l'œil du cœur $^{29}$ ", et Bernardin de Sienne ( "Tu'l miri coll'occhio del cuore $\left.{ }^{30} »\right)$, dans un sens qu'il restreint, toutefois, à l'amour filial.

\section{Images réelles et images mentales : des outils performatifs}

6 Je passe au second point pour lequel j'ai sélectionné divers exemples illustrant l'emploi rhétorique que Bernardin fait des images sensibles ou de celles mentales qu'il suscite dans l'esprit de son auditoire ${ }^{31}$. Il s'agit d'un même procédé, recherchant cet effet d'enargeia dont il a déjà été question, d'évidence visuelle, en donnant à voir sous les yeux. Ce qui change, ce sont les supports. "Io il più delle prediche ch'io fo, le fo per le piazze $^{32}$ ", proclame Bernardin. L'environnement urbain immédiat - la place publique - fournit au prédicateur un matériau de première main, exploitable pour un 
public citadin. Bernardin, prêchant à Sienne en 1427 sur la constance dans la vertu, devant le Palais communal, prend cet exemple :

Se tu fussi salito in su la Torre [celle du Mangia, que le public a en face de lui] [...] e elli ti venisse uno pensiero di cascare e gittartene giù, e così [...] che tu ti gittasse; e come fusse gitattoti e tu gridasse e pentissiti del male che tu fai, dicendo: "Soccorritemi, soccorritemi, ché mi pento del male ch'io fo"; dico che questo pentere non ti vale: elli ti converrà cadere e crepare in terra ${ }^{33}$.

Que met-il sous les yeux ? La chute (imaginaire) depuis la cime de la tour (bien réelle), dont tout le public présent évalue la hauteur, et le plongeon vertigineux du corps s'écrasant sur les pavés de la place. L'image pourra fonctionner comme image active ou agissante - l'imago agens des arts de la mémoire, depuis Cicéron - qui réactivera non seulement celle de la chute mais aussi les mots et l'intention qu'il a véhiculés, à savoir le commentaire moral sur la chute du pécheur. Autre exemple : Bernardin prêche sur la bonne voie, et non la mauvaise, à prendre pour aller à Dieu. Il utilise la comparaison suivante, celle de quelqu'un qui, pour se rendre à Rome, sortirait de Sienne par la Porte Camollia qui mène à Florence au lieu de prendre par la Porta Nuova qui mène à Rome ${ }^{34}$. La référence à une réalité de la géographie urbaine connue de tous souligne l'erreur grossière et le but de la leçon morale : on s'égare sur le mauvais chemin et l'on s'éloigne de Dieu. Toujours sur cette Piazza del Campo, Bernardin veut convaincre de la nécessité qu'il y a pour lui de prêcher sur la morale du mariage. Il oriente les regards de l'auditoire vers la Fontaine Gaia comme le support d'une métaphore : "Se pure [quella fonte] fusse stata avelenata, non so' io tenuto a dirvelo, che voi non ne beiate, acciò che voi non siate avelenati? Certo $s^{35}$. » Rappelons qu'on puisait alors à cette fontaine l'eau potable : laisser les habitants s'empoisonner, ce serait pour le prédicateur comme se rendre coupable de non-assistance à couples en danger de péché.

Bernardin transporte volontiers ses références visuelles à l'extérieur du périmètre urbain, hors des murs de la cité, comme dans les exemples suivants. Sur les mots de Jean dans l'Apocalypse "une grande montagne ${ }^{36}$ », que Bernardin interprète comme l'« orgueil de l'esprit»: "[...] Superbia. Mons magnus: uno monte grandissimo, come la Montamiata $^{37}$.» Le mont Amiata sert de référence pour un public qui peut voir le sommet de cette montagne depuis Sienne par temps très clair. Lors d'un prêche à Florence pour la Semaine sainte de 1425, voulant éclairer l'image des quatre jardins aux fausses fleurs du monde trompeur, il se donne parmi l'auditoire un interlocuteur imaginaire au nom parlant de « Gioioso » :

Gioioso mio, andianne in sul monte Morello [mont à proximité de Florence d'où l'on découvre un ample panorama sur la ville], e non t'incresca ch'io ti voglio mostrare di colassù che si vede la Marina e Firenze, ed altre terre, e parecchi belle cose. Or oltre andiamo, ed eccoti in sul monte. Or comincia a guardare Firenze. Vedilo tu? Sì, bene ${ }^{38}$.

De cette vue panoramique, il passe à un plan rapproché sur la maison d'un riche marchand. En apparence, tout y reflète le bonheur de l'opulence et d'une maisonnée nombreuse, mais en regardant plus attentivement :

Ora sta' a vedere. Che vedi? Vedo la moria che vi s'è appiccata; vedo morire il maggior figliuolo. Che vedi altro? Vego i figliuoli tutti morire, vego lui amalato, vego la roba cominciarsi a furare. [...] Io il veggio morto, la roba sbaragliata [... ${ }^{39}$.

Le regard se porte ensuite plus loin, vers la côte :

Guarda alla mancina, Gioioso mio, che vedi? Vego una nave venire al Porto Pisano collo vele piene e col vento in poppa, ella mi pare una gran nave. Che vedi altro? Vegola [piena] di mercatanzia [...]. Ista' a vedere. Che vedi? Io vego venire una 
fortuna dalla lunga, o'me! [...] vego la nave abbandonata e dà in iscoglio; ella si fende tutta, l'acqua sormonta; ella affonda con tutta la ciurma e la mercatanzia ${ }^{40}$.

11 Tout le passage est rythmé par les vedi, vedo, guarda : la visualisation est parfaitement réussie. "Mondo cieco!» («monde aveugle!») commente le «Joyeux », ou plutôt Bernardin, à propos d'un monde d'hommes qui ne savent pas voir au-delà des apparences une réalité instable, aveuglés par l'image sensible et fallacieuse du bonheur matériel : "Non è altro, questo mondo, che faccia e [fecc]ia: bella faccia di fuori e feccia di $d\left[\right.$ entro ${ }^{41} "$, " il traditore mondaccio ti mostra pure le c[ose pros]pere, il mele di sopra al veleno ${ }^{42} »$. Car l'œil de chair est englué dans le sensible, et l'âme peine à le dépasser, autre thème bonaventurien : "les images sensibles [phantasmata] enténèbrent l'âme et ne la laissent pas faire retour sur elle par l'intelligence ${ }^{43} »$, lit-on dans l'Itinéraire. «Voir», c'est donc aussi dépasser l'image sensible et l'apparence des choses pour atteindre la réalité qu'elles obscurcissent : «Tu hai veduto e vedi in questo mondo: tale ha stato grandissimo, e poi n'è cacciato e messo al basso [...]. Non comprendi tu essare grandi l'onde del mare di questo mondo ${ }^{44}$ ? » L'image, la chose vue doit permettre de passer du singulier à l'universel, faire ainsi expérience pour le présent : d'où le passage du « hai veduto » au « vedi $»$.

12 Parmi les ressources du langage figuré, Bernardin exploite peu les paraboles, même si, dit-il : "Gesù, fontana dell'eloquenza, usava sempre parabole ed esempi palpabili per far comprendere il Regno del cielo ${ }^{45}$. " Il n'est pas toujours sûr que son public en saisisse le sens : «Io non so se tu m'intendesti l'altro dì il mio parlare parabolico? " C'est pourquoi il préfère fonder ses comparaisons sur des images empruntées au quotidien. Mais il faut noter qu'il utilise ce que la Rhétorique à Herennius nomme les «similitudes frappantes » (" notatas similitudines $\left.{ }^{46} »\right)$, qui peuvent être le plus durablement fixées dans la mémoire, car c'est là le but poursuivi par le prédicateur : que les images soient si bien gravées dans la mémoire qu'elles puissent y réactiver les paroles auxquelles elles sont associées. D'où le choix d'images inattendues ${ }^{47}$ ou surprenantes, dont voici quelques échantillons. Prêchant sur l'honnêteté dans le mariage, il annonce à l'assemblée :

Domane [...] udirai in che peccati noi entraremo, che v'entrarò dentro come gallo in feccia. Vedeste mai il gallo quando entra in feccia? Elli v'entra dentro tutto pulito, coll'ale assetate in alto, per non imbrattarle, per potere volare a sua posta. Così farò io ${ }^{48}$.

13 Le fumier, ce sont les péchés des maris et des épouses qu'il va admonester le lendemain. Une image « contre toute attente » suffit parfois à fixer un discours développé sur la vie religieuse : «La religione è [una bocca piena di] denti $i^{49}$. » Un autre jour, Bernardin, à partir du passage de l'Apocalypse $(9,1-3)$ - qui est déjà en soi un texte accumulant les visions, transmises par le procédé de l'image et de la représentation ${ }^{50}$ - commente le mystère du mal, mal déclenché par la cinquième sonnerie de trompette et qui, sorti du puits de Satan, se répand comme les sauterelles sur la terre. Bernardin va transformer l'image symbolique et quelque peu opaque des criquets dévoreurs en une comparaison concrète - «l'essempio in pratica ${ }^{51} »-$ entre les insectes et les guerres ravageant les cités : "Io vi vo' dire il vero: egli me ne le par vedere adosso! Le locuste so' i manigoldi di Dio, che mettono a fine le vendette per giustizia ordinata da Dio. Sai chi so'? So' le genti dell'arme, e quali non fanno mai altro che danno ${ }^{52}$. » Bernardin décrypte cette image initiale et l'actualise en produisant à partir d'elle d'autres images qui doivent frapper l'imagination du public par la violence des actions qu'elles suggèrent :

Ellino [questi soldati] spogliano le donne e le fanciulle, e sforzano e vituperanle. [...] ardeno case, guastano palagi, guastano l'arti, distruggono i mestieri [...]. Di fuore de 
le terre prendono bestiame, pigliano uomini e donne, fannone strazio, martorianli, cavan lo' i denti $[. . .]^{53}$. relier ce lointain à une réalité proche et connue : «Vedesti mai come vola la contriemola che va saltellando, o come fa el franguello o la gazara? Vanno sempre saltando. Così fanno queste locuste di questi soldati $[. . .]^{54}$. » On assiste à un télescopage d'images dont ressort celle des nuées de soldats sautant et s'abattant d'une ville à l'autre pour tout y ravager.

\section{Médiation de deux images : la croix et la tablette}

Le livre de Lina Bolzoni, La rete delle immagini, est une étude irremplaçable sur l'utilisation des images peintes dans la prédication médiévale, et en particulier chez Bernardin de Sienne. Je ne reprendrai donc pas ce qu'elle dit des références aux fresques d'Ambrogio Lorenzetti au Palais communal de Sienne, mais je reviendrai brièvement sur la fameuse tablette du Nome di Gesù dont elle analyse la fonction ${ }^{55}$ et dont parle également Daniel Arasse dans son ouvrage ${ }^{56}$. J'ajouterai quelques mots sur un autre support du prédicateur, la croix ou l'image du Crucifié. La tablette est attachée à jamais à la figure de Bernardin, à son image telle qu'elle sera diffusée après sa mort. Il en est le créateur, même s'il n'est pas l'inventeur de la dévotion au Nom de Jésus, et commence à en faire usage dans sa prédication dès $1418^{57}$. La tablette consiste en trois lettres, abréviation de "Iesous" utilisée par les copistes grecs et passée telle quelle - YHS ou IHS - en latin ${ }^{58}$ : quoiqu'il en soit, il s'agit d'un christogramme, monogramme ou trigramme, peint en doré sur fond d'azur, à l'intérieur d'un soleil à douze rayons principaux et le plus souvent des rayons intermédiaires. Carrée ou ronde, la tablette peinte est montrée au public comme une ostension et Bernardin en fait une glose sur la base des quatre sens de l'Écriture ${ }^{59}$ (il voit ainsi dans le trigramme la Trinité). Bernardin a bien conçu sa tablette comme une image de type mnémotechnique ${ }^{60}:$ "Però fu ordinato per santa chiesa che si facesse la figura del Nostro Signore Gesù Cristo [...] acciò che vedut [o] con gli occhi corporali ti mettessi in memoria e alla mente il Signore $[. . .]^{61} . "$ On accède à une lecture de ces trois lettres sur le mode de signes imagés renvoyant à des réalités de foi. Ainsi, lors de sa prédication de 1425 à Florence, Bernardin explique : "Guarda ora $h$. Significa Spirito Santo, perché ingravidò Maria per Ispirito Santo; e vedi che la h è come una donna pregna ${ }^{62}$. " Bernardin fait de ces lettres des icônes qui serviront à mémoriser les articles de la foi, et le trigramme a pour lui valeur d'une icône du Christ : «Se [...] si concede il figurare Gesù per l'Agnus Dei o per l'agnello, adunque perché non si dee concedere il figurarlo nelle proprie parole del suo nome ${ }^{63}$ ? » L'image sensible et matérielle, en visualisant le nom de Jésus, le rend présent au plus intime de l'esprit et du cœur : «Veggendolo [il Nome] spesso coll'occhio corporale, el mostrerai all'occhio mentale di dentro, e spesso el nominerai per riverenza, per amore, per fede e pigliera'lo in abito, informa che sempre [...] Gesù, arai in cuore e in lingua $[. . .]^{64}$. » Bernardin ne dit pas autre chose en commentant le "Jésus » de l'Ave Maria : «E però vi dico che solamente questo nome suo, per lo suo amore, che voi il portiate nel cuore, nella mente e nel ventre ${ }^{65}$. "

Cette tablette fonctionne aussi comme une «enseigne [du Christ]» («un'insegna ») : Francesco Bruni interprète ainsi la tablette comme l'« enseigne sacrée » que Bernardin oppose à celles révérées par les factions, guelfes et gibelins : le sacré contre le profane ${ }^{66}$. J'ajouterai le culte de latrie contre l'idolâtrie et, en citant Bernardin, Dieu contre diable, image du Vrai Dieu contre les idoles des faux dieux de la politique: "L'idolatria è 
rinnovata; imperò che ora è entrato il diavolo in queste insegne de le parti [...]. E come allora [i diavoli] erano adorati in quelle statue, così ora so' adorati in queste insegne che tengono $i$ cristiani $^{67}$. "

17 Je terminerai par l'image de la croix dont il est peu parlé dans cette scénographie. Certaines représentations contemporaines montrent Bernardin exposant le crucifix à l'assemblée. On sait que la tablette fut violemment critiquée par ceux qui y voyaient un substitut à la croix. Dans son mémoire de 1427, l'augustin Andrea Biglia demande à Bernardin : « Pour quelle raison as-tu exposé [arboré] cette inscription de préférence à l'image du Christ en croix ${ }^{68}$ ? " Cette accusation rejoint celle d'un autre augustinien, Andrea da Cascia : «Il a aboli la croix du Christ ${ }^{69}$. » Or, le crucifix est une image à la fois réelle et mentale ${ }^{70}$. Prêchant sur la préparation à la communion, Bernardin utilise une parabole : un homme a été assassiné, mais sa veuve a gardé sa chemise ensanglantée pour inviter ses enfants à ne pas se venger et à faire la paix avec les coupables. L'Église, dit-il, est cette veuve: "Ella mostra la camicia insanguinata, la croce, il crucifisso Gesù, vestigia del vero crucifisso, accio che, quando il vedi in figura, abbi in orrore il peccato già commesso $^{71}$.» La parabole illustre bien l'emploi de ces imagines agentes, images agissantes, dont nous parlions plus haut. Quelle marchandise rend-elle riche ? demande encore Bernardin, quel est le trésor de Dieu? Le prédicateur recourt à l'image frappante et inattendue, susceptible de se graver dans l'esprit, celle d'une boutique :

Quale è questa buttiga? È il grazioso corpo di Cristo in croce, che à cinque fenestre

che sono le ferite de le mani, e de' pièi, e del costato, che, per amore di te, gittano

sangue, per lavare le tue colpe. La insegna di questa bottiga è la croce ${ }^{72}$.

Ici, nous avons l'image sensible par excellence, la Croix, image inscrite par ailleurs dans le trigramme même, sous la forme d'une barre horizontale coupant celle verticale de la lettre h et symbolisant visuellement la croix. C'est ce qu'explique Bernardin : «Guarda le lettare, dov'è scritto Iesù, o dove è la croce. [...] Non vedi tu il Crocifisso? - Sì. - Perché fu fatto? Perché tu ti ricordi come esso fu morto per te ${ }^{73}$. "

Pour conclure, il me semble que la diversité des images utilisées par Bernardin comme rhétorique de la persuasion est bien établie, même si ma sélection n'en rassemble qu'un faible échantillon. Sur cet aspect, encore peu exploré, des sermons de Bernardin, l'étude, dans un corpus immense, des images récurrentes comme de leurs variantes, pourrait se révéler utile pour dégager l'originalité et l'efficacité de leur utilisation par le prédicateur. Si leur importance tient certainement à une culture visuelle développée par l'Église pour une piété plus accessible que le texte, elle réside aussi dans leur capacité et leur efficacité à passer de la réalité visible aux réalités invisibles, comme le dit Bernardin lui-même : "Quando io vi predico, dimostrandovi talvolta, per essempro, de le cose visibili, e lo intelletto alza su alto a le cose invisibili ${ }^{74}$. »

\section{BIBLIOGRAPHIE}

ARASSE Daniel, Saint Bernardin de Sienne. Entre dévotion et culture : fonctions de l'image religieuse au XVe siècle, Paris, Hazan, 2014. 
Bolzoni Lina, La rete delle immagini. Predicazione in volgare dalle origini a Bernardino da Siena, Turin, Einaudi, 2002.

BouHAÏK-GIRONÈs Marie et Polo DE BEAULIEU Marie Anne (dir.), Prédication et performance du XII $a u$ XVI e siècle, Paris, Classiques Garnier, 2013.

BRUNI Francesco, La città divisa. Le parti e il bene comune da Dante a Guicciardini, Bologne, Il Mulino, 2003.

CARDINI Franco, Nel Nome di Gesù. Bernardino da Siena e la Battaglia mistica, Città di Castello, Il Cerchio, 2012.

Muzzarelli Maria Giuseppina, Pescatori di uomini. Predicatori e piazze alla fine del Medioevo, Bologne, Il Mulino, 2005.

Polo DE BEAUlieu Marie Anne et Berlioz Jacques, Images et prédication, dans J. Baschet et P.-O. Dittmar (dir.), Les images dans l'Occident médiéval, Turnhout, Brepols, 2015, p. 379-388.

WETZEL René et FLÜCKIGER Fabrice (dir.), Die Predigt im Mittelalter zwischen Mündlichkeit, Bildlichkeit und Schriftlichkeit. La prédication au Moyen Âge entre oralité, visualité et écriture, Zürich, Chronos Verlag, 2010.

\section{NOTES}

1. Bernardino da Siena, Prediche volgari sul Campo di Siena, 1427, éd. C. Delcorno, Milan, Rusconi, 1989, vol. I, p. 533, predica XVIII, désormais abrégé en Siena, 1427. (« Les exemples de ce que l’on voit touchent davantage que les mots que l'on dit.») Parmi les études générales, nombreuses, consacrées à la prédication médiévale, citons: L. Bolzoni, La rete delle immagini. Predicazione in volgare dalle origini a Bernardino da Siena, Turin, Einaudi, 2002 ; M. G. Muzzarelli, Pescatori di uomini. Predicatori e piazze alla fine del Medioevo, Bologne, Il Mulino, 2005; C. Delcorno, L'exemplum multiforme di Bernardino da Siena. Tra fonti scritte e canali di informazione, dans G. Baffetti, G. Forni, S. Serventi et O. Visani, "Quasi quidam cantus». Studi sulla predicazione medievale, Florence, Leo S. Olschki, 2009, p. 203-241; R. Wetzel et F. Flückiger (dir.), Die Predigt im Mittelalter zwischen Mündlichkeit, Bildlichkeit und Schriftlichkeit. La prédication au Moyen Âge entre oralité, visualité et écriture, Zürich, Chronos Verlag, 2010; M. Bouhaïk-Gironès et M. A. Polo de Beaulieu (dir.), Prédication et performance du XII ${ }^{e}$ au XVI ${ }^{e}$ siècle, Paris, Classiques Garnier, 2013.

2. Bernardin cite approximativement («Plus movent exempla quam verba ») Grégoire le Grand, Homiliarum In Ezechielem Prophetam Libri Duo (Homélies sur Ézéchiel), lib. II, homil. vII, 3 (P.L. LXXVI, p. 1014) : "Plus enim plerumque exempla quam ratiocinationis verba compungunt" (« En effet, la plupart du temps les exemples piquent davantage que les mots d'un raisonnement »).

3. À propos des images verbales dans le sermon ad populum, voir F. Flückiger et R. Wetzel, « Image verbale et prédication écrite. Transmission de la foi et enseignement des pratiques religieuses dans les sermons eucharistiques d'Engelberg ", dans Le Moyen Âge, tome CXVIII, ${ }^{\text {os }} 3-4,2012$, p. 545-579: «Performance orale par excellence, la prédication médiévale est aussi le lieu privilégié de l'“image verbale". Afin de transmettre son message, le prédicateur enrichit son sermon d'exempla, de récits imagés, de métaphores et d'allégories, qui permettront à l'auditoire de visualiser l'enseignement qui lui est destiné. Ces images immatérielles, qui suscitent autant d'images mentales dans l'esprit du destinataire alors qu'il écoute le prêche, jouent un rôle central dans la prédication. » (p. $545 ;<$ https://doi.org/10.3917/rma.183.0545>)

4. Rhétorique, III, X-XI. Voir B. Rougé, " "La (per così dire) energia delle figure”. Transporter, figurer, ou l'enargeia/energeia des œuvres : pour une énergétique de l'art », Aisthesis, <http:// 
dx.doi.org/10.13128/Aisthesis-10996> ; Id., « Le tableau efficace. Réflexions sur la performativité de la peinture (religieuse) », dans A. Dierkens, G. Bartholeyns et T. Golsenne (dir.), La performance des images, Bruxelles, Éditions de l'Université de Bruxelles, 2010, p. 364-365.

5. Ars Poetica, v. 180-181 : «Segnius inritant animos demissa per aurem / quam qua sunt oculis subiecta fidelibus [...]. »

6. J'emprunte à saint Thomas d'Aquin et à sa philosophie de la connaissance : «Ensuite [...] lorsqu'on cherche à connaitre intellectuellement quelque chose, on se forme par manière d'exemples des images dans lesquelles on regarde, pour ainsi dire, ce qu'on désire connaître. Également, quand nous voulons faire comprendre une chose à quelqu'un, nous lui donnons des exemples dont il puisse se former des images pour comprendre [“[...] proponimus ei exempla, ex quibus sibi phantasmata formare possit ad intelligendum"]. " (Somme théologique, Ia, q. 84, a. 7, Paris, Éditions du Cerf, vol. 1, p. 739)

7. Vita prima (deuxième partie, chap. 4 , § 97), dans Saint François d'Assise, Documents. Écrits et premières biographies, éd. T. Desbonnets et D. Vorreux, Paris, Éditions franciscaines, 1981, p. 276, note 2. Le texte latin dit: «[...] et non minus exemplo quam verbo cedificans audientes, de toto corpore fecerat linguam. "

8. Saint François, ouvr. cité, Vita secunda, chap. 157, § 207, p. 497.

9. Sur les « images actives ", que nous évoquons plus loin, voir l'ouvrage indispensable de F. A. Yates, L'art de la mémoire, Paris, Gallimard, coll. « Bibliothèque des histoires », 1975.

10. Siena, 1427, ouvr. cité, vol. I, p. 189 (IV) : « Tu dois te montrer attentif à entendre, presque sans broncher, en regardant bien le visage du prédicateur, et ne pas laisser ton œil se distraire de-ci de-là, mais le tenir fixé sans regarder ailleurs. » Précisons que le tutoiement est employé par saint Bernardin, comme avant lui le dominicain Giovanni Dominici, pour toucher plus directement le public, en alternance avec le voi.

11. Somme théologique, ouvr. cité, Ia, q. 84, a. 7, p. 739 : « Notre intelligence, dans l'état présent de la vie [...] ne peut avoir d'activité sans recourir à des images. »

12. Ibid., Ia, q. 78, a. 3 : "Visus autem [...] est maxime spiritualis, et perfectior inter omnes sensus, et communior. »

13. Siena, 1427, ouvr. cité, vol. I, p. 477 (xvI) : « Avec l'exemple, tu me comprendras. Bonaventure dit : "Plus movent visa, quam audita". On garde mieux à l'esprit ce qu'on voit que ce qu'on entend. » Pour la citation de saint Bonaventure, voir Sententiarum, III, d.24, dub.2.

14. San Bernardino da Siena, Le prediche volgari, Firenze, 1425, éd. C. Canarozzi, Florence, Libreria Editrice Fiorentina, 3 vol., 1940, vol.1, p.173: "Voir est plus profitable et vaut mieux qu'entendre, car la vertu visive [faculté de vision] est placée en plus haut lieu que l'ouïe. »

15. Siena, 1427, ouvr. cité, vol. 1, p. 477, sermon XVI: "Ces choses, vous les voyez continuellement. »

16. Ibid., vol. 1, p. 106, I : « Tous les anges l'entourent [...] comme tu le vois là-bas peint à la Porte Camollia. »

17. Ibid., vol. 1, p. 87, I : « Marie [...] monte là où, si tu l'examines attentivement, tu la verras couronnée comme une vraie impératrice [...]. »

18. Ibid., vol. 1, p. 123, II : « Va voir dans la Genèse au premier chapitre [...]. »

19. Ibid., vol. 2, p. 1000, xxxv : «Voyons-les, voulez-vous, plus en détail ?»

20. F. Yates, ouvr. cité, p. 20-22.

21. Siena, 1427, ouvr. cité, vol. 1, p. 135, II : « Là où tu vois dans ces mots sept propriétés de ceux qui veulent vivre selon le bien. »

22. Mc 7, 34.

23. Siena, 1427, ouvr. cité, vol. 1, p. 350, XI : «Ô Sienne, ouvre les yeux, ouvre les oreilles pour entendre et comprendre et voir ton salut !»

24. Ibid., vol. 1, p. 413, XIv : « Ouvre-toi, ouvre l'œil de l'intelligence, ouvre les oreilles. » 
25. Ibid., vol. 1, p. 105, I : « Je crois que tu as pu la voir avec ton intelligence monter vers la gloire, invitée par tous les esprits bienheureux [...]. »

26. Saint Augustin, La Genèse au sens littéral (De Genesi ad litteram), traduction, introduction et notes par P. Agaësse et A. Solignac, Paris, Institut d'études augustiniennes, p. 351 : «La première, nous l'appellerons vision corporelle (corporale) [...]. La seconde, nous l'appellerons vision spirituelle (spirituale) [...]. La troisième, nous l'appellerons vision intellectuelle (intellectuale), vision par l'intelligence. » (XII, VI, 15)

27. Saint Bonaventure, Itinéraire de l'esprit vers Dieu, introduction, traduction et notes par H. Duméry, Paris, Vrin, 1994, p. 31 (chap. I, § 4) : « Notre âme exerce trois principaux regards : le premier sur les corps extérieurs [...] le deuxième en elle-même et sur elle-même [...] le troisième sur le transcendant, d'où l'appellation d'esprit [intelligence]. »

28. Éph. $1,18$.

29. Saint Bernard, Livre du précepte et de la dispense, XIV, 36-38: "interior oculus ", " oculum cordis ». 30. Siena, 1427, ouvr. cité, vol. 2, p. 1211 (XLI) : « Tu le regardes avec l'œil du cœur. »

31. Sur la culture visuelle au Moyen Âge et ses liens avec la prédication, je renvoie à l'ouvrage de R. Wetzel et F. Flückiger, et à l'article de F. Flückiger et R. Wetzel, cités en note 1, ainsi qu'à M. A. Polo de Beaulieu et J. Berlioz, "Images et prédication », dans J. Baschet et P.-O. Dittmar, Les images dans l'Occident médiéval, Turnhout, Brepols, 2015, p. 379-388.

32. Siena, 1427, ouvr. cité, vol. 1, cit., p. 726, xxv : «La plupart de mes prêches, je les fais sur les places publiques. »

33. Ibid., vol. 2, p. 781-782, XXVII : "Si tu étais monté sur la Tour [...] et qu'il te venait l'idée de tomber et de te jeter en bas de cette tour, et [...] que tu jettes en bas ; et alors que tu te serais jeté et que tu crierais et que tu te repentirais du mal que tu fais, en disant: "Secourez-moi, secourezmoi, car je me repens du mal que je fais"; je dis que ce repentir ne te vaut rien : il te faudra tomber et t'écraser par terre. »

34. Ibid., vol. 1, p. 179, IV.

35. Ibid., vol. 1, p. 575, xx : «Cependant, si elle avait été empoisonnée, ne suis-je pas tenu de vous dire que vous n'en buviez pas, pour que vous ne soyez pas empoisonnés?»

36. Ap 8, 8-9.

37. Siena, 1427, ouvr. cité, vol. 1, p. 443, Xv : «Orgueil. Mons magnus : une très grande montagne, comme le Mont Amiata. »

38. Firenze, 1425, ouvr. cité, vol. 3, LVII, p. 248 : «Mon Joyeux, allons-nous en sur le mont Morello. Et n'en sois pas contrarié, car de là-haut je veux te montrer qu'on a une vue sur la mer et sur Florence et sur d'autres villes et sur plusieurs belles choses. Or donc, allons, et te voici sur le mont. Maintenant, commence par regarder Florence. Est-ce que tu la vois ? Oui ? Bien. » Dans une version (hélas!) "modernisée ", le même texte dans San Bernardino da Siena, Prediche della Settimana Santa. Firenze, 1425, éd. M. Bartoli, Milan, edizioni Paoline, 1995, p. 129-130.

39. Ibid., p. 249 : «Maintenant, vois. Que vois-tu? Je vois la mort qui s'y est attachée; je vois mourir le fils aîné. Que vois-tu d'autre ? Je vois mourir tous les enfants, je le vois lui, malade, je vois qu'on commence à voler ses biens. [...] Je le vois mort, tous ses biens dispersés [...]. »

40. Ibid., p. 249-250 : «Regarde à gauche, mon Joyeux, que vois-tu? Je vois un navire venir vers le port de Pise, voiles gonflées et le vent en poupe, on dirait un grand navire. Que vois-tu d'autre? Je le vois plein de marchandise [...]. Regarde bien. Que vois-tu ? Je vois venir de loin une tempête, hélas ! [...] je vois le navire abandonné et il va s'échouer sur un écueil ; il se brise entièrement et l'eau pénètre de partout ; et il coule avec tout l'équipage et toute la marchandise. »

41. Ibid., p. 244-245 : «Ce monde n'est rien d'autre qu'apparence et fumier : belle apparence audehors et fumier au-dedans.»

42. Ibid., p. 242 : «Ce traître et vilain monde te fait voir aussi les choses sous un jour prospère, le miel sur le poison.» 
43. Itinéraire..., ouvr. cité, p. 73 (chap. IV, § 1).

44. Siena, 1427, ouvr. cité, vol. 1, p. 351, XI : « Tu as vu et tu vois dans ce monde : un tel a un grand état, et puis il en est chassé et mis en bas [...]. Ne comprends-tu pas que les vagues de la mer qu'est ce monde sont grandes. "

45. Ibid., vol. 1, p.590, xx: "Jésus, fontaine de l'éloquence, usait toujours de paraboles et d'exemples palpables pour faire comprendre le Royaume des Cieux. »

46. Rhétorique à Herennius, III, XXXVII, 1 : «Imagines igitur nos in eo genere constituere oportebit, quod genus in memoria diutissime potest haerere. Id accidet, si quam maxime notatas similitudines constituemus. »

47. Voir ibid., I, x, $2:$ « praeter expectationem » (« contre toute attente »).

48. Siena, 1427, ouvr. cité, vol. 1, p. 547-548, XIX : «Demain [...] tu verras dans quels péchés nous entrerons, car j'y entrerai comme le coq dans le fumier. Avez-vous jamais vu le coq quand il entre dans le fumier? Il y entre tout propre, les ailes bien relevées pour ne pas les salir, pour pouvoir voler à sa guise. Je ferais pareil. »

49. Firenze, 1425, ouvr. cité, vol. 3, p. 315, LX : « La religion est une bouche pleine de dents. »

50. J. Giblet, "Introduction», dans L'Apocalypse. Une lecture commentée, Bruxelles, Culture et Vérité, 1997.

51. Siena, 1427, vol. 1, p. 334, x : « L'exemple concret. »

52. Ibid., vol. 2, p. 951, XxxIII : «Je veux vous dire la vérité : j'ai l'impression de les voir sur moi ! Les locustes sont les tortionnaires de Dieu, qui exécutent les vengeances selon une justice ordonnée par Dieu. Qui sont-elles, le sais-tu? Ce sont les gens d'armes qui ne font rien que des dégâts. »

53. Ibid., p. 951 : «[Ces soldats] dépouillent femmes et jeunes filles de leurs vêtements, et les forcent et les déshonorent [...] ils incendient les maisons, endommagent les palais, endommagent les arts, détruisent les métiers [...]. Hors des villes, ils s'emparent du bétail, prennent hommes et femmes, les torturent, les martyrisent, leur arrachent les dents [...]. »

54. Ibid., p. 953 : « As-tu jamais vu comment vole la bergeronnette qui va sautillant, ou le pinson ou la pie ? Ils se déplacent toujours en sautant. Et c'est pareil pour ces locustes de soldats [...]. »

55. L. Bolzoni, La rete delle immagini, ouvr. cité, p. 206-217.

56. D. Arasse, Saint Bernardin de Sienne. Entre dévotion et culture: fonctions de l'image religieuse au $X V^{e}$ siècle, Paris, Hazan, 2014. L'ouvrage rassemble des études parues précédemment. L'introduction et les trois premiers chapitres traitent de la tablette.

57. «[...] nel 1418 [...] all'Aquila, la mostra al popolo» (La rete delle immagini, ouvr. cité, p. 207) ; à Milan, en 1418, selon C. Delcorno, "L'ars prcedicandi di Bernardino da Siena », Lettere italiane, vol. XXXII, nº 4, 1980, p. 444.

58. Autorisant ainsi l'acronyme Iesus Hominum Salvator: voir L. Bolzoni, ouvr. cité, p. 239-240 (note 182), et F. Bruni, La città divisa. Le parti e il bene comune da Dante a Guicciardini, Bologne, Il Mulino, 2003, p. 354-358. Dans l'encadré, on lit: «In nomine Yesu omne genu flectatur, celestium, terrestrium et infernorum » [ "afin qu'au nom de Jésus tout genou fléchisse au ciel, sur terre et aux enfers »] (Phil. 2, 10).

59. San Bernardino da Siena, Le prediche volgari. Predicazione del 1425 in Siena, éd. C. Cannarozzi, Firenze, Rinaldi, 1958, vol. 2, p. 188-196 (predica Xxxv, Questa è la seconda predica del nome di Gesù). Repris par D. Arasse, Saint Bernardin de Sienne, ouvr. cité, p. 41-43.

60. Sur ces « immagini per ricordare », voir S. Serventi, « Le immagini mnemotecniche nelle lettere di direzione spirituale. Girolamo da Siena », dans R. Wetzel et F. Flückiger (dir.), Die Predigt im Mittelalter, ouvr. cité, p. 197-222.

61. Cité (d'un sermon de 1425 à Florence) par P.G. Pesce, «La religiosità popolare nella predicazione bernardiniana », dans L. Glinka (dir.), L'evangelizzazione in san Bernardino da Siena. Saggi e ricerche, Rome, Ateneo Pontificio Antoniano, 1980, p. 76-77 : «C'est pourquoi il fut ordonné 
par la sainte Église que l'on fasse une représentation figurée de Notre Seigneur Jésus Christ [...] afin que, vu par les yeux corporels, elle mette le Seigneur dans ta mémoire et dans ton esprit [...].»)

62. Siena, 1425, ouvr. cité, vol. 2, p. 191, Xxxv : « Regarde à présent le h. Il signifie l'Esprit saint, car Marie conçut de l'Esprit saint ; et tu vois que le h est comme une femme enceinte. »

63. Cité par L. Bolzoni, ouvr. cité, p. 217 (Siena, 1425, ouvr. cité, vol. 1, p. 172, x) : « Si [...] l’on permet de représenter Jésus par l'Agnus Dei ou par l'agneau, pourquoi donc ne doit-on pas le représenter dans les mots mêmes de son nom?»

64. San Bernardino da Siena, Le prediche volgari, éd. C. Cannarozzi, Pistoia, Libreria Editrice Fiorentina, vol. 2, p. 208, XL: «En le voyant [le Nom] souvent avec ton œil corporel, tu le montreras à ton œil mental du dedans, et tu diras souvent son nom par révérence, par amour, par foi et tu t'en revêtiras de manière à ce que toujours [...] Jésus, tu l'auras dans ton cœur et sur la langue [...]. »

65. Siena, 1427, ouvr. cité, vol. 2, p. 846, XxIX : «C'est pourquoi je vous dis de porter son nom seul, en raison de son amour, dans le cœur, dans l'esprit et dans le ventre. »

66. F. Bruni, La città divisa, ouvr. cité, p. 350-353.

67. Siena, 1427, vol. 1, p. 468, XVI : «L'idolâtrie est renouvelée, car à présent le diable est entré dans ces enseignes des partis [...]. Et, de même qu'autrefois les diables étaient adorés dans les statues, de même à présent ils sont adorés dans ces enseignes que portent les chrétiens. »

68. Cité par F. Bruni, ouvr. cité, p. 370, d'après Gaiffier : «Et quidem te oro quam rationem habueris ut potius eam scripturam quam ipsum Yhesu Christi crucifixi signum proponeres?»

69. Ibid., p. 370, d'après Longpré : « crucem Christi evacuavit ».

70. Voir N. Bériou, « De la lecture aux épousailles. Le rôle des images dans la communication de la Parole de Dieu au XIII ${ }^{\mathrm{e}}$ siècle », Cristianesimo nella storia, vol. XIV, nº 3, 1993, p. 535-568.

71. Firenze, 1425, ouvr. cité, vol. 3, p. 394, LXIII : «Elle montre sa chemise ensanglantée, la croix, Jésus crucifié, empreinte du vrai crucifié, pour qu'en le voyant en image, tu aies en horreur le péché commis. »

72. Siena, 1425, ouvr. cité, vol. 1, p. 3, I : «Quelle est cette boutique ? C'est le gracieux corps du Christ en croix, qui a cinq fenêtres qui sont les blessures des mains, et des pieds, et du côté, et qui, par amour pour toi, jettent du sang pour laver tes fautes. L'enseigne de cette boutique, c'est la Croix. »

73. Siena, 1427, ouvr. cité, vol. 2, p. 919, XxxII : « Regarde les lettres, où est écrit Jésus, ou là où est la croix. [...] ne vois-tu pas le crucifix ? - Si. - Pourquoi a-t-il été fait ? Pour que tu te souviennes comment il est mort pour toi. »

74. Ibid., vol.2, p. 1302, XLIII: "Quand je prêche pour vous, en vous montrant parfois, par l'exemple, des choses visibles, et l'intelligence s'élève en haut vers les choses invisibles [...].

\section{RÉSUMÉS}

Dans les sermons de Carême de Bernardin de Sienne, l'emploi d'images matérielles ou sensibles, dites aussi "corporelles", et d'images immatérielles ou mentales revêt une très grande importance. Elles contribuent en effet à l'efficacité du discours homilétique. Cette étude aborde trois aspects majeurs : la rhétorique visuelle chez le prédicateur, la fonction performative des images réelles et des images mentales, et, outre de nombreux exemples textuels, examine 
également deux images significatives associées à la prédication de Bernardin (la tablette du Nom de Jésus et la Croix).

Nelle prediche di Quaresima di Bernardino da Siena, l'uso delle immagini materiali o sensibili, o cosiddette «corporali», e delle immagini immateriali o mentali riveste una grandissima importanza, in quanto esse contribuiscono all'efficacia del discorso omiletico. Questo studio affronta tre aspetti rilevanti: la retorica visiva del predicatore, la funzione performativa delle immagini reali e di quelle mentali, ed oltre a numerosi esempi testuali, esamina anche due immagini significative associate alla predicazione di Bernardino (la tavoletta del Nome di Gesù e la Croce).

In the sermons of Lent of Bernardine of Siena, the use of material or sensitive images (also called "bodily") and immaterial or mental images, is of great importance. They contribute to the effectiveness of the homiletic discourse. This study focuses on three major aspects: the preacher's visual rhetoric, the performative function of real images and mental images, and, in addition to numerous textual examples, also examines two significant images associated with Bernardin's preaching (the tablet with the Divine Name and the Cross).

\section{INDEX}

Parole chiave : Bernardino da Siena, immagini sensibili, immagini mentali, predicazione Mots-clés : Bernardin de Sienne, images sensibles, images mentales, prédication

Keywords : Bernardino da Siena, sensitive images, mental images, preaching

\section{AUTEUR}

\section{SERGE STOLF}

Université Grenoble Alpes, LUHCIE 